\title{
The Effect of Thermocycling on Interfacial Bonding Stability of Self-Etch Adhesives: OCT Study
}

\author{
T. A. Bakhsh $\mathbb{D D}^{1,2}$ and A. Turkistani $\mathbb{D}^{1}$ \\ ${ }^{1}$ Department of Restorative Dentistry, Faculty of Dentistry, King Abdulaziz University, P.O. Box 80209, Jeddah 215-89, Saudi Arabia \\ ${ }^{2}$ Cariology and Operative Dentistry Department, Tokyo Medical and Dental University, 1-5-45 Yushima, Bunkyo-ku, \\ Tokyo 113-8549, Japan
}

Correspondence should be addressed to T. A. Bakhsh; taabakhsh@kau.edu.sa

Received 20 January 2021; Revised 20 April 2021; Accepted 24 May 2021; Published 9 June 2021

Academic Editor: Li Wu Zheng

Copyright (C) 2021 T. A. Bakhsh and A. Turkistani. This is an open access article distributed under the Creative Commons Attribution License, which permits unrestricted use, distribution, and reproduction in any medium, provided the original work is properly cited.

\begin{abstract}
Objective. The aim of this study was to monitor the behavior of interfacial gaps formed under different bonded polymeric restorations before and after thermocycling (TC), using swept-source optical coherence tomography (SS-OCT) and confirming the obtained findings with confocal laser scanning microscopy (CLSM). Materials and Methods. Cylindrical class I cavities were prepared in twenty noncarious human premolar teeth $(1.5 \mathrm{~mm}$ depth $\times 3.5 \mathrm{~mm}$ diameter $)$ and divided randomly into two groups: TS and SN, according to the adhesive system $(n=10)$. In the TS group, one-step self-etch adhesive Clearfil Tri-S Bond Plus (Kuraray Noritake Dental, Japan) was used, followed by composite restoration using Estelite Sigma Quick (Tokuyama Dental, Japan). In the SN group, the cavities were restored with the two-step self-etch/composite silorane-based resin restoration system (3M ESPE, USA). All specimens were restored in bulk filling technique and cured in accordance with the manufacturers' instructions. Both groups were imaged under SS-OCT after $24 \mathrm{~h}$ and recorded as controls. Then, each group was subjected to thermal challenge using the TC machine $\left(5-55^{\circ} \mathrm{C}\right)$ and B-scans were recorded at different TC intervals $(2600,5200$, and 10000$)$. In order to confirm the SS-OCT findings, additional specimens were prepared, scanned, and sectioned for CLSM observation. Results. B-scans demonstrated white clusters at the tooth-resin interface that corresponded to the gap location on CLSM images. The TS group showed significantly less gap formation than the SN group before and after TC $(p<0.001)$. Conclusions. An optimal composite adaptation can be achieved when the bonded restoration comprises a combination of an adhesive containing 10-MDP monomer and a considerable highly filled composite.
\end{abstract}

\section{Introduction}

Adhesive dentistry has been continuously developed, attempting to simplify the restorative procedure and reducing resin polymerization shrinkage as well. Many of the newly introduced materials showed satisfactory immediate sealing results. However, the durability of sealed interfaces over time is still a challenge. In clinical situations, the restoration is subjected to mechanical and thermal stresses of the oral cavity, challenging the long-term durability of the adhesive layer and the composite adaptation [1-3]. Bond durability is critical for the long-term success of composite restoration, especially in restorations with dentin margins
[4-6]. To anticipate the long-term performance of newly introduced materials, aging by simulating stressful oral conditions in vitro or water storage has been used $[7,8]$. One of the main methods utilized in adhesive tests is thermal cycling or thermocycling (TC) [9]. Dental restorations in the oral cavity are subjected to TC because of the intermittent temperature changes as a result of the day-night cycles [8]. Usually, the adhesive layer subjected to TC undergoes cycles of high and low stresses at different temperatures [10]. This is attributed to the apparent differences in the thermal expansion coefficient of adhesive and bonded substrates. Consequently, damage and debonding may occur due to the residual stresses in the polymeric layer, which are generated 
over time as a result of the viscoelastic response. This would reduce bond durability and occasionally lead to restoration failure [10].

Optical coherence tomography (OCT) is a wellestablished imaging system in biomedical fields [11]. In dentistry, OCT has been an attractive tool for monitoring and long-term studies [12-14]. The reliance on backscattered light from tissue structure to produce real-time images has allowed high-resolution subsurface evaluation without destructing the specimen. OCT has been successfully used to monitor dental restoration and the progression of demineralization or remineralization of lesions [15-20].

The objective of the study was to monitor the behavior of interfacial gaps formed under different bonded polymeric restorations before and after TC using swept-source OCT (SS-OCT) and confirm the obtained findings with confocal laser scanning microscopy (CLSM). The null hypothesis was that the percentages of interfacial gaps in bonded restorations with one-step and two-step self-etch adhesives do not change after TC.

\section{Materials and Methods}

2.1. Tested Materials. The two adhesive systems used in this study were one-step self-etch adhesive Clearfil Tri-S Bond Plus (TP; Kuraray Noritake, Japan) and two-step self-etch silorane adhesive system (SA; 3M ESPE, USA). Two dental composites were used in this study: Estelite Sigma Quick (EQ; Tokuyama Dental, Japan) and Filtek Silorane composite (FS; 3M ESPE, USA). The chemical compositions of the used materials and method of application are listed in Table 1.

2.2. Specimen Preparation. The study was approved by the Research Ethics Committee of King Abdulaziz University (code: 068-16), which is in accordance with the guiding principles for investigational methods found in the Declaration of Helsinki of the World Medical Association. Based on a pilot study, sample size calculation was made using a 0.05 alpha value and $80 \%$ power to detect a difference of $25 \%$ (PiFace, http://homepage.stat.uiowa.edu/ rlenth/Power/). The common standard deviation within a group was assumed to be $18 \%$. The estimated sample size for each group should be at least 9 [21]. As a result, twenty extracted noncarious human premolar teeth were finally selected and preserved for 2 weeks in distilled water at $4^{\circ} \mathrm{C}$ before beginning the experiment. The roots were removed at the cementoenamel junction, and the remaining crown was cleansed from tissue debris and calculus. After a slight reduction of the cusps, cylindrical class I cavities were prepared on the occlusal surface $(1.5 \mathrm{~mm}$ depth $\times 3.5 \mathrm{~mm}$ diameter $)$ and the cavity floor being located in dentin. Each specimen was placed in a vial and given a code. Then, they were distributed equally into two groups: TS and SN. This was carried out using simple random sampling technique, which involves equal and random distribution of the specimens into two experimental groups.

In the TS group, the prepared cavities were bonded with the TP adhesive, photoactivated for $10 \mathrm{~s}$, and restored with the EQ composite that was light cured for $10 \mathrm{~s}$. The SA adhesive and the FS composite were used to restore the cavities of the SN group. The light curing time of the SA adhesive was $10 \mathrm{~s}$ while it is $20 \mathrm{~s}$ for the FS composite restoration. All materials were applied and photoactivated according to the manufacturers' recommendations (Table 1) using a halogen curing unit (Optilux501, Kerr, USA; $500 \mathrm{~mW} / \mathrm{cm}^{2}$ intensity).

In order to maintain the reproducibility of the restoration process, material application and photoactivation were carried out according to the previously described protocol in prior studies $[15,22,23]$. After preparing the cavities, each specimen was held vertically to avoid pooling the adhesive to one side of the cavity during the bonding procedure. Before dispensing the adhesive bond into the mixing well, the adhesive bottle was shaken to ensure complete binding of the adhesive components. Then, using a disposable microbrush, the adhesive was applied in a rubbing motion. The airdrying step was performed while a 3-way syringe was held vertically over the bonded cavity at a $20 \mathrm{~cm}$ distance to remove the excess adhesive, obtain a uniform adhesive layer, and ensure the solvent's evaporation [24]. Photoactivation of the applied adhesive was accomplished while the light guide tip of the light cure was positioned within a $2 \mathrm{~mm}$ distance from the occlusal surface and continuous irradiation was performed. Next, a small volume of the composite was squeezed out of the composite tube and covered by a plastic dappen dish to protect the composite from premature polymerization. Afterward, a plastic filling instrument was used to fill the prepared cavity with one increment of dental composite in bulk filling technique. After compacting the composite and removing the excess, each specimen's occlusal surface was covered with a glass slide to standardize the packing pressure and improve composite structural integrity while being photopolymerized. Composite photoactivation was conducted similar to the above-mentioned adhesive lightcuring protocol. The light-guide tip of the light cure was positioned within $2 \mathrm{~mm}$ distance from the restoration surface, and continuous irradiation was performed. These aforementioned steps were strictly followed to standardize the restorative procedure in both groups and ensure the adequacy of the assessed outcome.

Afterward, all restored specimens were kept in distilled water for $24 \mathrm{~h}$ before SS-OCT baseline scanning. A schematic illustration of the research methodology is presented in Figure 1.

2.3. SS-OCT Imaging and Thermocycling. The technical specification of the SS-OCT imaging system (IVS2000, Santec, Japan) is described thoroughly elsewhere [23, 25]. Initially, each specimen was placed on a $3 \mathrm{D}$ micrometer stage. The occlusal surface was positioned parallel to the floor and perpendicular to the projected laser beam under the SS-OCT probe. Twelve sequential 2D scans (B-scans) were obtained at every $250 \mu \mathrm{m}$ for each specimen in the mesiodistal direction and considered baseline scans.

After that, all specimens were subjected to $\mathrm{TC}$ at $5^{\circ} \mathrm{C}$ and $55^{\circ} \mathrm{C}$ in a water bath for 2600,5200 , and 10000 cycles with a transfer time of $5 \mathrm{~s}$ and a dwell time of $30 \mathrm{~s}$. Sequential B-scans were obtained for both groups at the end of 
TABLE 1: List of the composition of the used materials in this study.

\begin{tabular}{|c|c|c|c|c|}
\hline $\begin{array}{l}\text { Material (Manufacturer) } \\
\text { Code }\end{array}$ & Composition & Lot number & Fillers \% & Manufacturer recommendation \\
\hline $\begin{array}{l}\text { Clearfil Tri-S Bond Plus } \\
\text { adhesive } \\
\text { One-step self-etch } \\
\text { (Kuraray Noritake } \\
\text { Dental, Japan) } \\
\text { TP }\end{array}$ & $\begin{array}{l}\text { Adhesive: MDP, Bis-GMA, HEMA, } \\
\text { hydrophilic aliphatic dimethacrylate, } \\
\text { hydrophobic aliphatic methacrylate, colloidal } \\
\text { silica, sodium fluoride, dl-camphorquinone, } \\
\text { accelerators, initiators, ethanol, water, others } \\
(\mathrm{pH}=2.3)\end{array}$ & 00007A & - & $\begin{array}{l}\text { Adhesive } \\
\text { (i) Apply bond for } 10 \mathrm{~s} \\
\text { (ii) Dry with mild pressure air flow for } \\
5 \mathrm{~s} \\
\text { (iii) Light cure for } 10 \mathrm{~s}\end{array}$ \\
\hline $\begin{array}{l}\text { Estelite Sigma Quick } \\
\text { Universal composite } \\
\text { (Tokuyama Dental, } \\
\text { Japan) } \\
\text { EQ }\end{array}$ & $\begin{array}{c}\text { Composite: Bis-GMA, TEGDMA, silica- } \\
\text { zirconia fillers, silica-titania fillers, } \\
\text { photoinitiators. }\end{array}$ & J018 & $\begin{array}{l}82 \% \text { (wt.) } \\
71 \% \text { (vol) }\end{array}$ & $\begin{array}{l}\text { Composite } \\
\text { (i) Light cure the composite for } 10 \mathrm{~s} \text {, } \\
\text { keeping the curing light tip within a } \\
\text { distance of } 2 \mathrm{~mm} \text { from the surface }\end{array}$ \\
\hline \multirow{2}{*}{$\begin{array}{l}\text { Silorane system adhesive } \\
\text { Two-step self-etch } \\
\text { (3M ESPE, USA) } \\
\text { SA }\end{array}$} & $\begin{array}{l}\text { Primer: phosphorylated methacrylates, } \\
\text { Vitrebond copolymer, Bis-GMA, HEMA, } \\
\text { water, ethanol, silane-treated silica filler, } \\
\text { initiators, stabilizers (pH: } 2.7 \text { ). }\end{array}$ & N289224 & - & $\begin{array}{l}\text { Primer } \\
\text { (i) Apply primer for } 15 \mathrm{~s} \\
\text { (ii) Gentle air dry } \\
\text { (iii) Light cure for } 10 \mathrm{~s}\end{array}$ \\
\hline & $\begin{array}{l}\text { Bond: hydrophobic dimethacrylate, } \\
\text { phosphorylated methacrylates, TEGDMA, } \\
\text { silane-treated silica filler, initiators, stabilizers. }\end{array}$ & N209848 & - & $\begin{array}{l}\text { Bond } \\
\text { (i) Apply bond } \\
\text { (ii) Gentle air dry } \\
\text { (iii) Light cure for } 10 \mathrm{~s}\end{array}$ \\
\hline $\begin{array}{l}\text { Filtek Silorane } \\
\text { Low shrinkage posterior } \\
\text { composite } \\
\text { (3M ESPE, USA) } \\
\text { FS }\end{array}$ & $\begin{array}{l}\text { Composite: silorane resin, CQ, iodonium salt, } \\
\text { electron donor, quartz filler, yttrium fluoride, } \\
\text { stabilizers, pigments. }\end{array}$ & N204592 & $\begin{array}{l}76 \% \text { (wt.) } \\
55 \% \text { (vol) }\end{array}$ & $\begin{array}{l}\text { Composite } \\
\text { (i) Light cure the composite for } 20 \mathrm{~s} \text {, } \\
\text { keeping the curing light tip within a } \\
\text { distance of } 2 \mathrm{~mm} \text { from the surface }\end{array}$ \\
\hline
\end{tabular}

HEMA: 2-hydroxyethyl methacrylate; Bis-GMA: bisphenol-A-diglycidyl methacrylate; TEGDMA: triethyleneglycoldimethacrylate; MDP: 10methacryloyloxydecyl dihydrogen phosphate; CQ: camphorquinone; wt: weight; vol: volume.

each TC interval (2600, 5200, and 10000 cycles). Each Bscan corresponded to an image $8 \mathrm{~mm} \times 6.6 \mathrm{~mm}$ dimensions (2001 pixels $\times 1019$ pixels) obtained in approximately $100 \mathrm{~ms}$.

2.4. OCT Image Analyses. Each B-scan was imported and managed by a plug-in macrofile coded in an image analysis software (ImageJ ver. 1.42q) in order to convert OCT data to a grayscale image according to the signal intensity value of each pixel in the matrix $[22,23,26]$. By analyzing the scans at the cavity floor, some areas showed high signal intensities that appeared as bright clusters of pixels, while others showed dark gray pixels. Based on previous reports, these bright clusters of pixels represent interfacial gaps [22, 23, 26]. OCT image analysis and gap quantification were carried out according to the protocol described by Bakhsh et al. [22, 26]. The imported OCT data was converted to an 8-bit grayscale image, subjected to a median filter (1px radius), and cropped to the area of interest, which included the whole cavity floor. Then, the image binarization function was employed to convert the cropped grayscale image to black and white image to determine the target pixels with significantly higher brightness compared to other dark pixels in the background. The transformed target pixels into black pixels on a white background at the cavity floor were considered as interfacial gaps, which were calculated in percentage according to the following equation:

$$
\begin{aligned}
& \text { Gap(bright clusters length) } \% \\
& \quad \cdot \frac{\text { total length of bright clusters at each slice }}{\text { length of the cavity floor at that slice }} \times 100 \text {. }
\end{aligned}
$$

2.5. CLSM Imaging. After 10000 cycles, the imaged specimens were trimmed with $\mathrm{SiC}$ papers (600-200 grit) and polished with diamond polishing films and pasts in a descending size order $(6.0-0.25 \mu \mathrm{m})$ followed by interfacial examination using CLSM. Additional specimens for both groups were prepared, restored, OCT scanned, and trimmed after $24 \mathrm{~h}$ before being polished and examined under CLSM.

2.6. Statistical Analysis. Statistical analysis of the results was performed using a statistical software package (SPSS-2 for Windows: SPSS, USA). The values obtained from B-scans of each section were averaged and included in the statistical analysis. As the distribution of data was normal, a parametric test was performed. The difference in mean gap percentage between the tested groups at different TC intervals (baseline, 2600, 5200, and 10000 cycles) with a significance level defined as alpha $=0.05$ was calculated.

\section{Results}

By analyzing SS-OCT images, some B-scans were showing high signal intensity (intense backscattered reflection) in the form of a bright cluster of pixels at the cavity floor at 


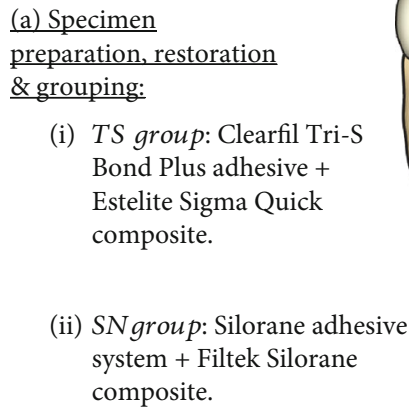

(i) TS group: Clearfil Tri-S Bond Plus adhesive + Estelite Sigma Quick composite.

(ii) SNgroup: Silorane adhesive system + Filtek Silorane composite.

\section{(d) OCT imaging:}

B-scans after each time interval $(2600,5200$, 10000 thermocycles) for TS \& SNgroups.
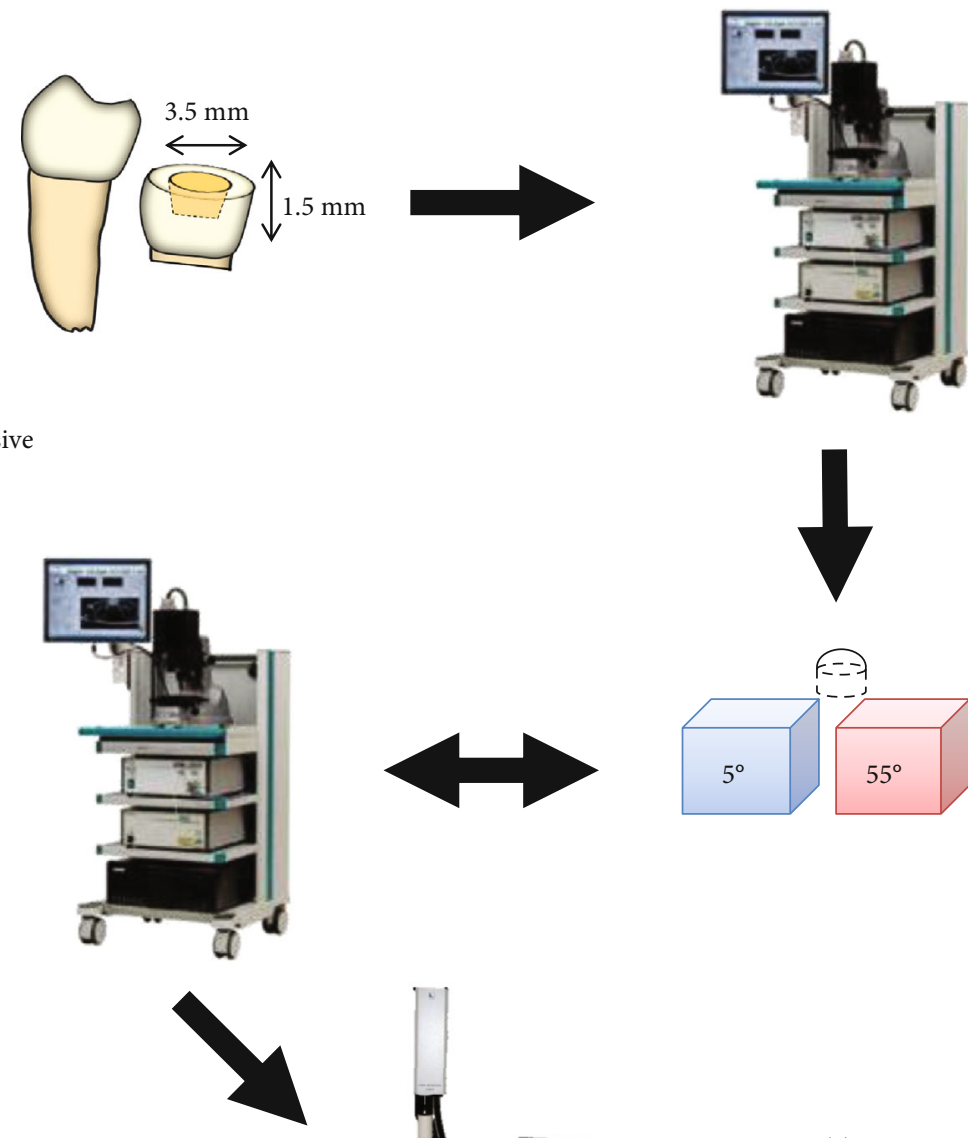

(b) OCT imaging: B-scans after $24 \mathrm{~h}$ for TS \& SN groups.
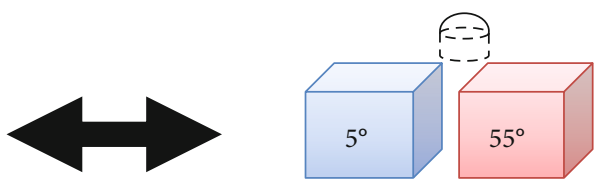

(c) Thermocycling:

(i) 2600

(ii) 5200

(ii) 10000 (e) CLSM imaging: Specimen trimming, polishing \& tooth-resin interface examination.

FIGURE 1: Schematic illustration showing the methodology of the study.

some regions (Figures 2 and 3). Other regions were not disclosing any abrupt changes in the pixel intensity values at the cavity floor, which corresponded to areas with no interfacial gaps between the bonded substrates under CLSM. Regions that showed high backscattered reflection under SS-OCT were confirmed as interfacial gap areas under CLSM (Figures 4 and 5).

A mixed ANOVA repeated measures with a GreenhouseGeisser correction showed that the mean gap percentage differed significantly at different TC intervals (within subjects) for each adhesive system $(p<0.001)$. In addition, the type of adhesive system had a significant effect (between subjects) on the mean gap percentages within each TC interval $(p<0.001)$.

Student's independent $t$-test had shown statistically significant differences between tested groups, TS and SN, at each time interval $(p<0.001)$ (Table 2). Before subjecting the specimens to TC (baseline), the gap percentage in TS was smaller than in SN, with mean gap percentages of 2.22 $(\mathrm{SD} \pm 1.78)$ and 20.55 (SD \pm 14.11$)$, respectively. After 2600 TC, the mean gap increased significantly in both groups, with a mean gap percentage of $3.03(\mathrm{SD} \pm 2.24)$ in the TS group and $53.86(\mathrm{SD} \pm 20.74)$ in the $\mathrm{SN}$ group. Then, the mean gap percentage of both groups decreased after 5200 and 10000 thermocycles, with mean gap percentages of 2.38 $(\mathrm{SD} \pm 1.6)$ and $2.92(\mathrm{SD} \pm 2.02)$ for the TS group and 31.6 $(\mathrm{SD} \pm 17.21)$ and $36.10(\mathrm{SD} \pm 16.64)$ for the $\mathrm{SN}$ group, respectively.

The estimated mean gap percentages at each time interval are given, as depicted in Figure 6.

\section{Discussion}

Thermocycling has been widely used in dental research as an aging technique. According to the International Organization for Standardization (ISO), the thermocycling regimen ISO/TS 11405 standard (2015) can be carried out while testing the quality of an adhesive bond between the restorative dental materials and the hard dental tissue. This includes the measurements from a tensile bond strength test, microleakage test, or marginal gap test. On the other hand, optical assessment of bonded composite restorations using OCT has been recently introduced to locate and quantify interfacial gaps in real time, which had altered the perception of polymerization shrinkage. Based on the current findings, TC is an appropriate method for understanding the durability of 


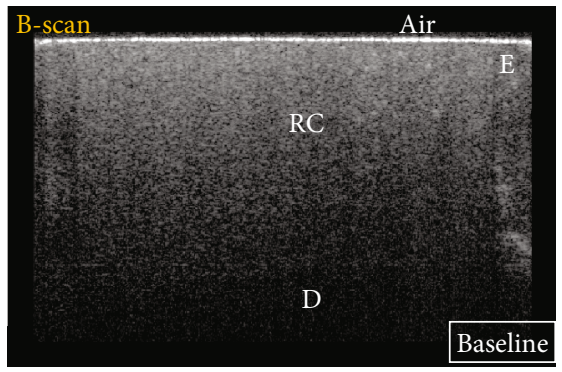

(a)

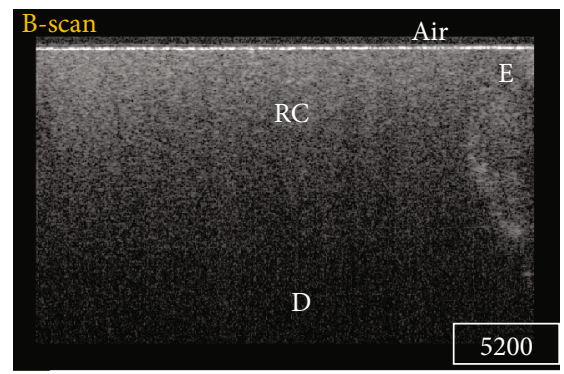

(c)

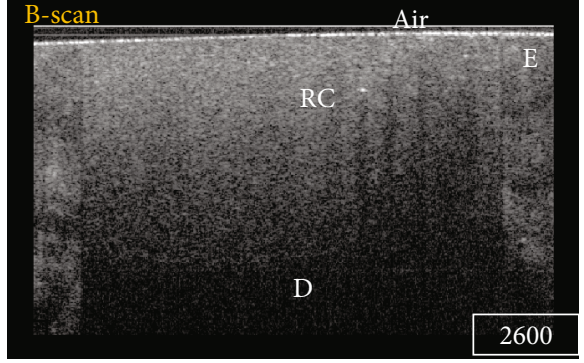

(b)

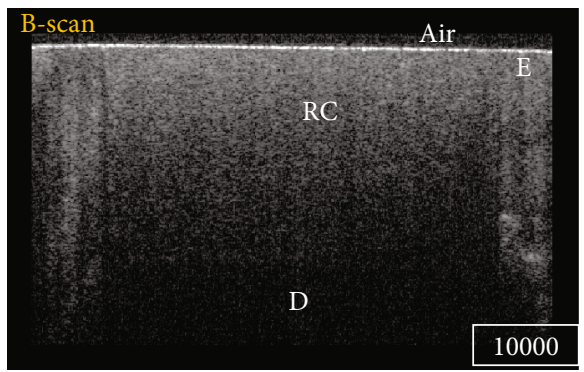

(d)

Figure 2: B-scan for a representative specimen of the TS group at different time intervals: (a) baseline, (b) 2600 TC, (c) 5200 TC, and (d) 10000 TC. Regardless of the time interval, the absence of bright bands of pixels with low backscattered reflection at the cavity floor indicates no loss of interfacial seal. E: enamel; D: dentin; RC: resin composite.

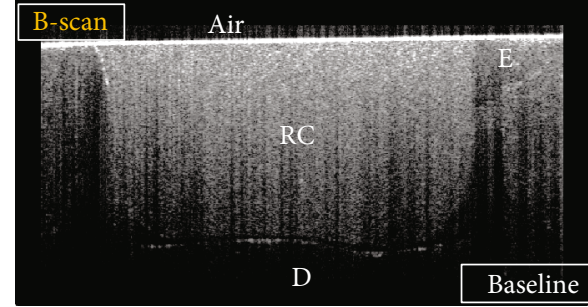

(a)

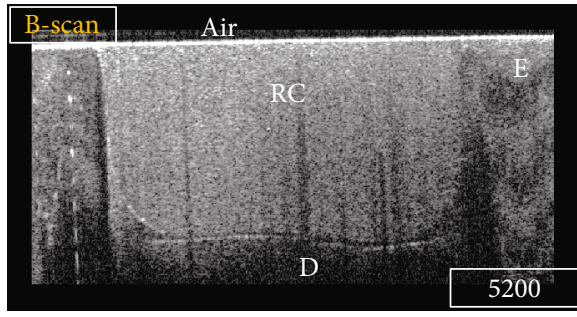

(c)

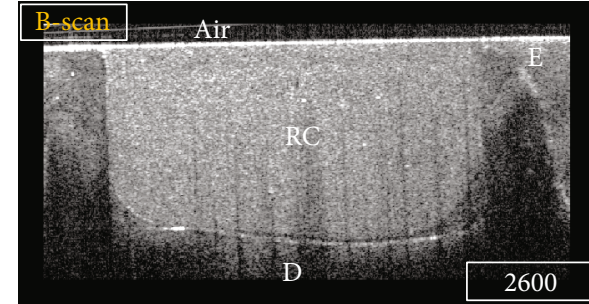

(b)

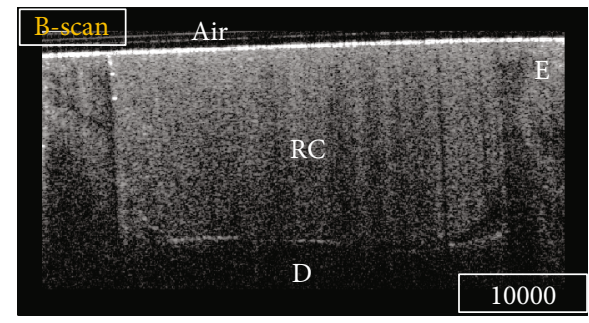

(d)

Figure 3: B-scan for a representative specimen of the SN group at different time intervals: (a) baseline, (b) 2600 TC, (c) 5200 TC, and (d) 10000 TC. Strong backscattered reflection at the cavity floor in some regions was demonstrated on the B-scan as bright bands of pixels that were considered an interfacial gap. In contrast, regions that did not show an increase in signal intensity at the tooth-resin interface indicated no loss of interfacial seal. E: enamel; D: dentin; RC: resin composite.

the bonded composite restorations. All bonding materials used in the present study with a TC method had clinically acceptable levels of marginal gaps.

In this study, SS-OCT was used to detect and monitor the changes in the gap length of bonded restorations subjected to thermal challenge. Gap detection by SS-OCT is based on analyzing the alteration of the backscattered signals that ascribed to the difference in the refractive indices $(n)[22,23]$. Previous reports described the sudden increase in the signal inten- sity at the tooth-resin interface of bonded restorations as microgaps and a loss of interfacial seal [23]. Usually, bright bands of white clusters at the cavity floor are produced in B-scans when the OCT light passes through dissimilar substrates with a divergent index of refractions called "Fresnel" phenomena. On the contrary, this phenomenon is undetectable when OCT light passes through substrates with comparable refractive indexes. The index of refractions of enamel and dentin is relative to dental composites, ranging from 


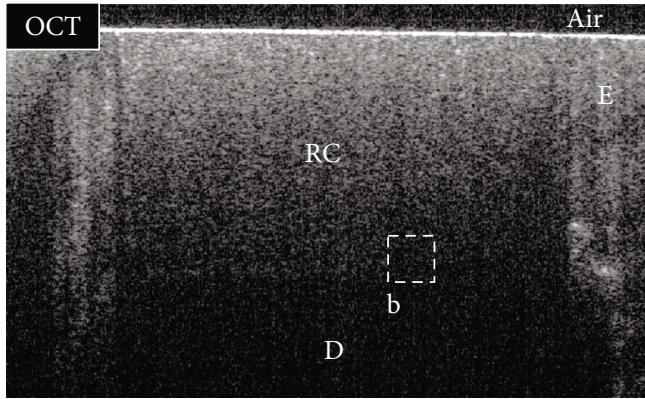

(a)

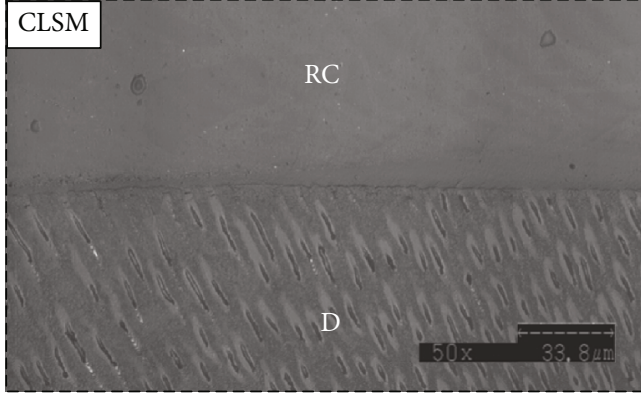

(b)

FiguRE 4: Representative images for the TS group. (a) B-scan showed low backscattered reflection at the cavity floor, including the target area (dotted box (b)). (b) A confirmatory CLSM image ( $\times 50$ magnification) for the target area (dotted box (a)) showed no interfacial gap at the dentin-resin interface. E: enamel; D: dentin; RC: resin composite.

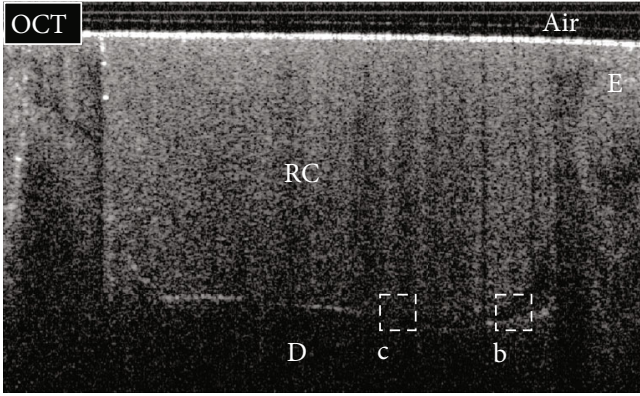

(a)

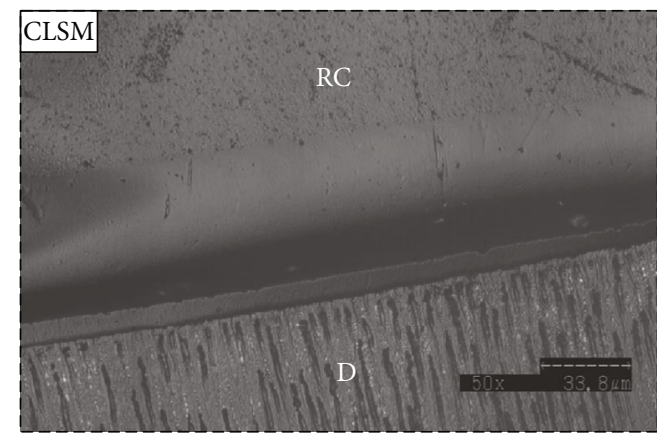

(b)

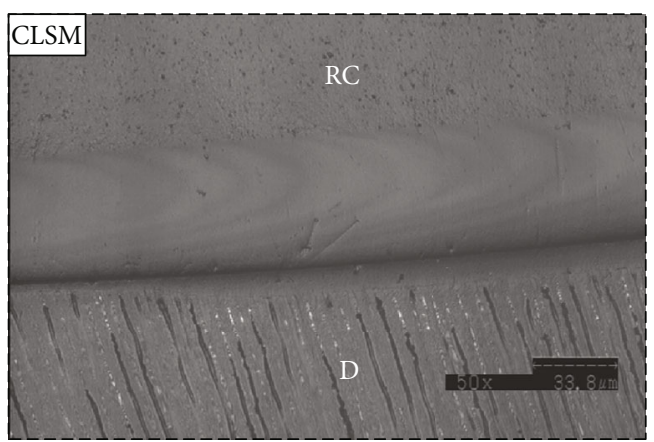

(c)

FIGURE 5: Representative images for the SN group $(\mathrm{a}-\mathrm{c})$. (a) B-scan showed low backscattered reflection at the cavity floor in some areas (dotted box (b)), while other areas showed intense backscattered reflection (dotted box (c)). (b, c) Confirmatory CLSM images $(\times 50$ magnification) corresponded to the obtained OCT findings. (b) The interfacial gap was detected within the adhesive layer and corresponded to the bright band of pixels in the OCT B-scan. (c) At the same time, it showed no interfacial gap in the dentin-resin interface of other areas, which was seen as dark pixels at the cavity floor in the presented OCT B-scan. E: enamel; D: dentin; RC: resin composite.

1.5 1.6 [23, 26-28]. Therefore, reduced signal interference will be detected on the signal intensity's profile (A-scan) in the absence of interfacial gaps. Regardless of the cause of the interfacial gaps, they are filled with either air or water, in a form of saliva or dentinal fluid, that have indexes of refractions equal to 1 and 1.3 , respectively [22, 27]. This means that signal interference at the tooth-restoration interface is more intense when voids or microgaps are filled with air than with water. In this regard, the difference in signal intensities between the tested groups can be justified at different time intervals.
Dental adhesives are composed of hydrophilic and hydrophobic components that are designed to act as stress absorbers between hard dental tissues and dental composites. Several factors affect the restorations' durability in the oral cavity, which include the type of the substrate being bonded, surface area, rate and duration of the thermal challenge, thermal and hygroscopic expansion coefficients of the bonded substrates, magnitude and duration of the masticatory forces, and presence of defects within bonded substrates [29-31]. The coefficient of thermal and hygroscopic expansion of the bonded resins influenced the results of the current study. 
TABLE 2: Mean gap percentage at each time interval using Student's independent $t$-test.

\begin{tabular}{lccccc}
\hline $\begin{array}{l}\text { Time interval } \\
\text { (thermocycles) }\end{array}$ & Group & Mean (\%) & SD & Std. error & $p$ value \\
\hline \multirow{2}{*}{ Baseline } & TS & 2.22 & 1.78 & 0.30 & \\
& SN & 20.55 & 14.11 & 2.35 & $<0.001$ \\
2600 & TS & 3.03 & 2.24 & 0.37 & \\
& SN & 53.86 & 20.74 & 3.46 & $<0.001$ \\
5200 & TS & 2.38 & 1.60 & 0.27 & \\
& SN & 31.60 & 17.21 & 2.87 & $<0.001$ \\
10000 & TS & 2.92 & 2.02 & 0.34 & \\
& SN & 36.10 & 16.64 & 2.77 & $<0.001$ \\
\hline
\end{tabular}

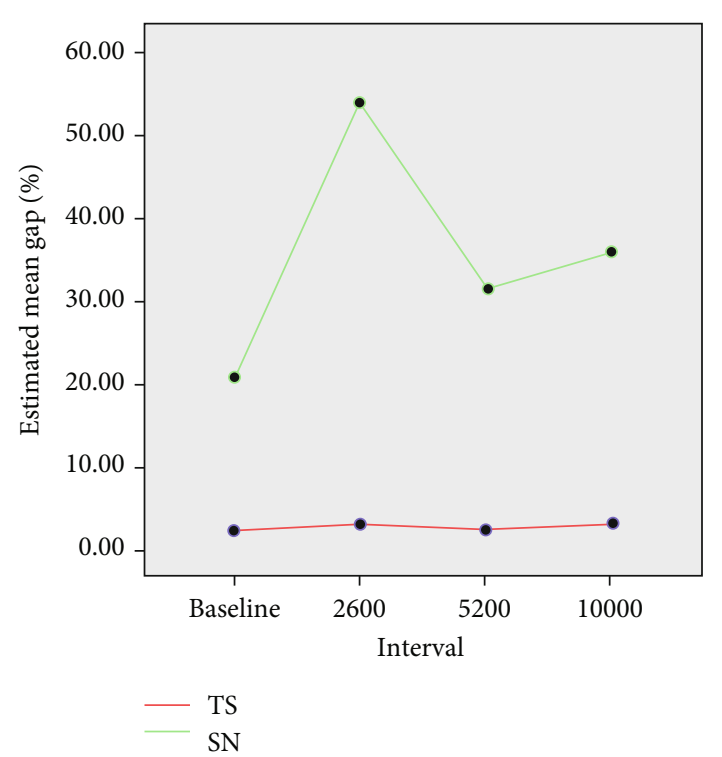

Figure 6: Comparison of estimated mean gap percentages at four time intervals between the TS and $\mathrm{SN}$ groups.

The results showed a divergence in gap measurements across the different time intervals. Before subjecting the specimens to TC, the mean gap percentage of TS and SN was equal to 2.22 and 20.55, respectively. After 2600 TC, the mean gap percentage had increased for both groups, which could be attributed to the generated interfacial stresses by thermal aging. Later, the gap in these bonded restorations had significantly decreased after 5200 and 10000 TC. Several studies showed that marginal integrity and bond strength of dental restorations decrease after short periods of TC and they ascribed their findings to the decrease in the resins' coefficient of thermal expansion [32-34]. However, other studies showed that prolonged thermal challenge would induce hygroscopic expansion in dental resin materials that improve the marginal integrity and compensate for the effect of polymerization shrinkage [35]. Both tested adhesives contain BisGMA and HEMA that may contribute to more water uptake and cause adhesive layer swelling, bond degradation under the influence of TC, which may explain the changes in the mean gap percentage in both groups over the different time intervals [36].
TP adhesive is a mild one-step self-etch adhesive $(\mathrm{pH}=2.3)$ that contains phosphate monomer (10-MDP), which is characterized by a stable chemical bonding to hydroxyapatites [22]. This adhesive in the TS group was bonded to EQ composite with a volumetric filler loading equal to $71 \%$. On the other hand, SA adhesive bonding system was bonded to FS composite in the SN group that was recognized as a low-shrinkage composite and contains siloxane core and four oxirane rings attached [37]. By examining the chemical composition of the two-step self-etch SA adhesive system, the adhesive primer with $\mathrm{pH}$ equals 2.7 contains a solvent, hydrophilic and hydrophobic monomers, fillers, initiators, and stabilizers. In contrast, the SA bond contains only hydrophobic monomers, fillers, and initiators. The SA adhesive layer was bonded to the FS composite that has a volumetric filler loading of around 55\%. Although the FS composite includes a silorane ring responsible for reducing the shrinkage and optimizing its properties, the reduced filler loading has jeopardized composite adaptation. This low filler content had indirectly affected adhesive stability to resist the generated contraction stress against the cavity walls compared to the EQ composite. Moreover, the low $\mathrm{pH}$ of TP adhesive in the TS group would produce deep resin tags and a wide hybrid layer, unlike in the SN group. Thus, the difference in the monomer acidity and composite filler loading has influenced the study results and explains the difference in the mean gap percentage of both groups across the different time intervals.

It is noteworthy that although several studies demonstrated high bond strength with two-step self-etch adhesives in comparison to one-step self-etch adhesives, the primer utilized in the SN group resembles the components and action of one-step self-etch adhesives [38-40]. Two studies showed some voids and blister formation within the adhesive and between silorane bond and composite layers. A possible explanation would be related partly to incomplete solvent evaporation and uncured monomers that remain on the adhesive surface and partly to the high viscosity of this bonding system, which may trap air voids during the air-thinning step of the adhesive $[38,41]$. These findings could explain the low performance of the SN group.

Within the limitations of the study, which include a narrow study design and a limited number of teeth-the null hypothesis was rejected.

\section{Conclusion}

An optimal composite adaptation can be achieved when the bonded restoration comprises a combination of an adhesive containing 10-MDP monomer and a considerable highly filled composite. Moreover, interfacial deformity under bonded composites can be monitored effectively using OCT.

\section{Data Availability}

Data are available on request upon contacting the corresponding author by email (taabakhsh@kau.edu.sa). 


\section{Conflicts of Interest}

There were no conflicts of interest.

\section{Acknowledgments}

This research was partly supported by Tokyo Medical and Dental University (Tokyo, Japan) and by King Abdulaziz University (Jeddah, Saudi Arabia). The authors are thankful to Prof. Junji Tagami, Dr. Yasushi Shimada, and Dr. Alireza Sadr for their tremendous technical support.

\section{References}

[1] D. C. Watts and A. J. Cash, "Kinetic measurements of photopolymerization contraction in resins and composites," Measurement Science and Technology, vol. 2, no. 8, pp. 788-794, 1991.

[2] A. J. Feilzer, A. J. De Gee, and C. L. Davidson, "Setting stress in composite resin in relation to configuration of the restoration," Journal of Dental Research, vol. 66, no. 11, pp. 1636-1639, 1987.

[3] E. Lutz, I. Krejci, and T. R. Oldenburg, "Elimination of polymerization stresses at the margins of posterior composite resin restorations: a new restorative technique," Quintessence International, vol. 17, no. 12, pp. 777-784, 1986.

[4] D. F. Rigsby, D. H. Retief, C. M. Russell, and F. R. Denys, "Marginal leakage and marginal gap dimensions of three dentinal bonding systems," American Journal of Dentistry, vol. 3, no. 6, pp. 289-294, 1990.

[5] F. Lutz, I. Krejci, B. Luescher, and T. R. Oldenburg, "Improved proximal margin adaptation of class II composite resin restorations by use of light-reflecting wedges," Quintessence International, vol. 17, no. 10, pp. 659-664, 1986.

[6] C. L. Davidson, A. J. de Gee, and A. Feilzer, "The competition between the composite-dentin bond strength and the polymerization contraction stress," Journal of Dental Research, vol. 63, no. 12, pp. 1396-1399, 1984.

[7] M. M. Abdel-Azim and H. M. Shaheen, "Punch shear strength of base cements. I. Effect of aging and water storage on polycarboxylate and zinc phosphate cements," Egyptian Dental Journal, vol. 21, no. 4, pp. 67-74, 1975.

[8] T. D. Larson and J. R. Jensen, "Microleakage of composite resin and amalgam core material under complete cast crowns," The Journal of Prosthetic Dentistry, vol. 44, no. 1, pp. 40-44, 1980.

[9] J. C. Jones, A. R. Grieve, and E. Harrington, "A machine for thermal cycling in the investigation of marginal leakage. Its development and use," British Dental Journal, vol. 146, no. 7, pp. 207-211, 1979.

[10] G. R. Humfeld and D. A. Dillard, "Residual stress development in adhesive joints subjected to thermal cycling," The Journal of Adhesion, vol. 65, no. 1-4, pp. 277-306, 1998.

[11] D. Huang, E. A. Swanson, C. P. Lin et al., "Optical coherence tomography," Science, vol. 254, no. 5035, pp. 1178-1181, 1991.

[12] M. M. Mandurah, A. Sadr, Y. Shimada et al., "Monitoring remineralization of enamel subsurface lesions by optical coherence tomography," Journal of Biomedical Optics, vol. 18, no. 4, article 046006, 2013.

[13] T. A. Bakhsh, A. S. Bakry, M. M. Mandurah, and M. A. Abbassy, "Novel evaluation and treatment techniques for white spot lesions. An in vitro study," Orthodontics \& Craniofacial Research, vol. 20, no. 3, pp. 170-176, 2017.

[14] J. Na, B. H. Lee, J. H. Baek, and E. S. Choi, "Optical approach for monitoring the periodontal ligament changes induced by orthodontic forces around maxillary anterior teeth of white rats," Medical \& Biological Engineering \& Computing, vol. 46, no. 6, pp. 597-603, 2008.

[15] T. A. Bakhsh, N. H. Altouki, L. S. Baeesa et al., "Effect of self-etch adhesives on the internal adaptation of composite restoration: a CP-OCT study," Odontology, vol. 107, no. 2, pp. 165-173, 2019.

[16] T. A. Bakhsh, A. A. Bakhsh, S. Bakhsh et al., "Effect of bioglass on artificially induced enamel lesion around orthodontic brackets: OCT study," in Lasers in Dentistry XXIV, pp. 1047302-1-1047302-9, San Francisco, California, USA, 2018.

[17] A. Turkistani, S. Nakashima, Y. Shimada, J. Tagami, and A. Sadr, "Microgaps and demineralization progress around composite restorations," Journal of Dental Research, vol. 94, no. 8, pp. 1070-1077, 2015.

[18] M. N. Luong, Y. Shimada, K. Araki, M. Yoshiyama, J. Tagami, and A. Sadr, "Diagnosis of occlusal caries with dynamic slicing of 3D optical coherence tomography images," Sensors, vol. 20, no. 6, p. 1659, 2020.

[19] A. A. Al Tuwirqi, E. A. El Ashiry, A. Y. Alzahrani, N. Bamashmous, and T. A. Bakhsh, "Tomographic Evaluation of the Internal Adaptation for Recent Calcium Silicate- Based Pulp Capping Materials in Primary Teeth," BioMed Research International, vol. 2021, Article ID 5523145, 10 pages, 2021.

[20] M. A. Abbassy, T. A. Bakhsh, and A. S. Bakry, "A novel evaluation method for detecting defects of the bonded orthodontic bracket-tooth interface," BioMed Research International, vol. 2021, Article ID 6634595, 8 pages, 2021.

[21] R. V. Lenth, "Statistical power calculations1," Journal of Animal Science, vol. 85, suppl_13, pp. E24-E29, 2007.

[22] T. A. Bakhsh, A. Sadr, Y. Shimada et al., "Concurrent evaluation of composite internal adaptation and bond strength in a class-I cavity," Journal of Dentistry, vol. 41, no. 1, pp. 60-70, 2013.

[23] T. A. Bakhsh, A. Sadr, Y. Shimada, J. Tagami, and Y. Sumi, "Non-invasive quantification of resin-dentin interfacial gaps using optical coherence tomography: validation against confocal microscopy," Dental Materials: Official Publication of the Academy of Dental Materials, vol. 27, no. 9, pp. 915-925, 2011.

[24] S. B. Werle, A. Steglich, F. Z. Soares, and R. O. Rocha, "Effect of prolonged air drying on the bond strength of adhesive systems to dentin," General Dentistry, vol. 63, no. 6, pp. 68-72, 2015.

[25] T. A. Bakhsh, "Optical comparison between micro-CT and OCT in imaging of marginal composite adaptation: observational study," Journal of Microscopy, vol. 282, no. 2, pp. 136145, 2021.

[26] T. A. Bakhsh, A. Sadr, Y. Shimada, A. Turkistani, R. Abuljadayel, and J. Tagami, "Does lining class-II cavities with flowable composite improve the interfacial adaptation?," Journal of Adhesion Science and Technology, vol. 34, no. 4, pp. 400-416, 2020.

[27] I. Hariri, A. Sadr, Y. Shimada, J. Tagami, and Y. Sumi, "Effects of structural orientation of enamel and dentine on light attenuation and local refractive index: an optical coherence tomography study," Journal of Dentistry, vol. 40, no. 5, pp. 387-396, 2012. 
[28] E. Z. Alsayed, I. Hariri, A. Sadr et al., "Optical coherence tomography for evaluation of enamel and protective coatings," Dental Materials Journal, vol. 34, no. 1, pp. 98-107, 2015.

[29] W. Zhou, S. Liu, X. Zhou et al., "Modifying adhesive materials to improve the longevity of resinous restorations," International Journal of Molecular Sciences, vol. 20, no. 3, 2019.

[30] C. K. Yiu, N. Hiraishi, F. R. Tay, and N. M. King, "Effect of chlorhexidine incorporation into dental adhesive resin on durability of resin-dentin bond," The Journal of Adhesive Dentistry, vol. 14, no. 4, pp. 355-362, 2012.

[31] A. A. Ali, H. A. El Deeb, O. Badran, and E. H. Mobarak, "Bond durability of self-etch adhesive to ethanol-based chlorhexidine pretreated dentin after storage in artificial saliva and under intrapulpal pressure simulation," Operative Dentistry, vol. 38, no. 4, pp. 439-446, 2013.

[32] M. S. Cenci, T. Pereira-Cenci, T. A. Donassollo, L. Sommer, A. Strapasson, and F. F. Demarco, "Influence of thermal stress on marginal integrity of restorative materials," Journal of Applied Oral Science, vol. 16, no. 2, pp. 106-110, 2008.

[33] A. Versluis, W. H. Douglas, and R. L. Sakaguchi, "Thermal expansion coefficient of dental composites measured with strain gauges," Dental Materials: Official Publication of the Academy of Dental Materials, vol. 12, no. 5-6, pp. 290-294, 1996.

[34] M. Miyazaki, M. Sato, H. Onose, and B. K. Moore, "Influence of thermal cycling on dentin bond strength of two-step bonding systems," American Journal of Dentistry, vol. 11, no. 3, pp. 118-122, 1998.

[35] Y. Momoi and J. F. McCabe, "Hygroscopic expansion of resin based composites during 6 months of water storage," British Dental Journal, vol. 176, no. 3, pp. 91-96, 1994.

[36] J. M. C. Lima, L. C. Anami, S. M. B. Pereira et al., "Dentin/composite bond strength: effect of aging and experimental unit," Journal of Adhesion Science and Technology, vol. 35, 2021.

[37] G. A. Maghaireh, N. A. Taha, and H. Alzraikat, "The siloranebased resin composites: a review," Operative Dentistry, vol. 42, no. 1, pp. E24-E34, 2017.

[38] T. A. Bakhsh, A. Sadr, and J. Tagami, "Focused ion beam processing for transmission electron microscopy of composite/adhesive interfaces," Journal of Adhesion Science and Technology, vol. 29, no. 3, pp. 232-243, 2015.

[39] P. H. P. D'alpino, N. C. de Farias, M. S. Silva, M. F. de Goes, A. H. M. González, and V. D. I. Hipólito, "Compatibility between silorane adhesive and simplified methacrylate-based adhesive systems," Dental Materials Journal, vol. 32, no. 2, pp. 263-273, 2013.

[40] A. Mine, J. De Munck, A. Van Ende et al., "TEM characterization of a silorane composite bonded to enamel/dentin," Dental Materials: Official Publication of the Academy of Dental Materials, vol. 26, no. 6, pp. 524-532, 2010.

[41] T. A. Bakhsh, A. Sadr, M. M. Mandurah, Y. Shimada, O. Zakaria, and J. Tagami, "In situ characterization of resindentin interfaces using conventional vs. cryofocused ionbeam milling," Dental materials, vol. 31, no. 7, pp. 833-844, 2015. 